\title{
Magnetic behavior of nanostructured Fe films measured by magnetic dichroism
}

\author{
K. W. Edmonds, C. Binns, ${ }^{\text {a) }}$ S. H. Baker, and S. C. Thornton \\ Department of Physics and Astronomy, University of Leicester, Leicester LEI 7RH, United Kingdom \\ P. Finetti \\ CLRC Daresbury Laboratory, Daresbury, Warrington, WA4 4AD, United Kingdom
}

(Received 7 February 2000; accepted for publication 13 June 2000)

\begin{abstract}
The magnetic properties of Fe nanostructured films have been studied using magnetic linear and circular dichroism in x-ray photoemission spectroscopy. The samples were prepared by the deposition of nanoscale Fe clusters, size 1-4 nm, onto thin $\mathrm{Cu}$ films. The linear dichroism, which is used to measure the in-plane magnetization, increases with increasing film thickness, with a sharp increase between 1 and 1.5 ML coverage. The circular dichroism, which measures the out-of-plane magnetization, is zero within the experimental error at all thicknesses studied. Capping an Fe film with an ultrathin Pd layer results in a factor of 3 decrease of the linear dichroism response. (C) 2000 American Institute of Physics. [S0021-8979(00)04518-7]
\end{abstract}

\section{INTRODUCTION}

Magnetism is strongly sensitive to atomic-scale changes in symmetry, coordination, and confinement. Nanostructured magnetic materials represent model systems for the study of such effects, and display novel phenomena such as a modified electronic structure and magnetic anisotropy, and enhanced orbital and spin magnetic moments. ${ }^{1-4}$ By forming nanoscale clusters prior to deposition onto a substrate, a narrow size distribution can be achieved, and nanostructured systems can be fabricated with a high degree of control and relatively few constraints. ${ }^{5}$

Magnetic dichroism in $\mathrm{x}$-ray photoemission spectroscopy (XPS) is a powerful tool for the study of nanostructured surfaces, probing the electronic structure and the magnetic properties simultaneously. ${ }^{6,7}$ The information provided is element specific and tunably surface sensitive. The effect can be observed using either linear ${ }^{8}$ or circular $^{9}$ polarized x-rays, and consists of a change in the lineshape and intensity of a core level photoemission spectrum upon reversing either the sample magnetization or the polarization of the light. The experiments are usually performed with a finite angular acceptance, and are referred to as either magnetic linear or circular dichroism in angular distributions of photoelectrons (MLDAD or MCDAD). Unlike the spin-resolved photoemission techniques, which suffer from the low efficiency of spin detection, magnetic dichroism measurements can be achieved with only a factor of 2 increase in the measurement time compared to spin-integrated XPS. MLDAD and MCDAD are not as powerful as magnetic X-ray circular dichroism (MXCD) in the photoabsorption by transition metal $L$ edges $^{3}$ since photoemission spectra must be measured with the magnetic field off, i.e., in remanence. They are, however, ultra-sensitive in situ probes of the magnetization in ultrathin films and in the experimental arrangement described here, where the geometry can be changed between measuring

${ }^{a)}$ Electronic mail: cb12@le.ac.uk in-plane and out-of-plane, are ideal tools for investigating anisotropy.

In this work, magnetic dichroism in XPS was used to characterize of $\mathrm{Fe}$ cluster-assembled films deposited on a polycrystalline $\mathrm{Cu}$ film of thickness $300 \AA$. MLDAD and MCDAD were used to measure the in-plane and out-of-plane remanent magnetizations, respectively, as a function of the film thickness. The effect of a Pd capping layer on the magnetic dichroism signal of the Fe films was also observed.

\section{EXPERIMENT}

The experiment was performed within an ultrahigh vacuum (UHV) chamber attached to beamline 5D of the SRS, Daresbury laboratory. The beamline delivers a usable photon flux across the energy range $20-180 \mathrm{eV}$, of either linear polarization or, by obstructing one half of the photon beam, up to $80 \%$ circular polarization of either helicity.

Substrates were prepared using a $\mathrm{Cu}$ vapor source to deposit $\mathrm{Cu}$ films of thickness $300 \AA$ A onto a Mo plate attached to a liquid He cooled cryostat within the UHV chamber. The $\mathrm{Fe}$ films were grown by directing a beam of nanoscale $\mathrm{Fe}$ clusters, produced using a high temperature gas-aggregation cluster source ${ }^{10}$ which was coupled to the UHV chamber through a gate valve, onto the substrate. The source produces a log-normal distribution of cluster sizes, with a peak at 2.2 $\mathrm{nm}$ and a median diameter of $3 \mathrm{~nm}$. Previous scanning tunneling microscopy studies have indicated that this size distribution is preserved upon deposition. ${ }^{11}$ The source is equipped with a quadrupole filter, which allows a narrow distribution of cluster sizes to be selected, ${ }^{4}$ although this was not used in the present experiment in order to minimize the deposition time.

The substrate temperature was maintained at $40 \mathrm{~K}$ during the film growth and throughout the measurements. During cluster deposition, the chamber pressure was $\approx 10^{-5}$ mbar due to the presence of the He bath gas used for aggregation of the $\mathrm{Fe}$ vapor, while the residual contaminants 
a) MCDAD applied field $\underset{\text { or }}{\longleftarrow}$

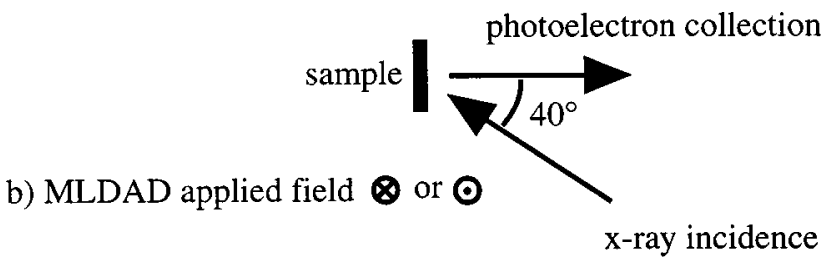

FIG. 1. Geometry of the experiment. The XPS measurements were obtained with either linearly polarized (MLDAD) or $80 \%$ circularly polarized (MCDAD) $x$-rays incident at $40^{\circ}$ to the surface normal with electrons collected along the surface normal. Remanent magnetization was either along the surface normal (MCDAD) or in the plane of the sample and perpendicular to the plane defined by the photon incidence and electron collection (MLDAD).

remained at the $10^{-11}-10^{-12}$ mbar level. After closing the gate valve between the chamber and the source, the vacuum recovered to better than $10^{-10}$ mbar within 2 min. The thickness of the deposited cluster films was monitored by ionizing a proportion of the beam and integrating the substrate drain current during deposition. The integrated current was calibrated using a quartz crystal thickness monitor. In the following the cluster film thickness is expressed in terms of a complete monolayer (ML) of closely packed $2.2 \mathrm{~nm}$ clusters.

A Pd capping layer was deposited on one sample using metal vapor source in UHV. The Pd layer thickness was estimated from the attenuation of the $\mathrm{Fe} 3 p$ intensity, and from the relative sizes of the $\mathrm{Fe} 3 p$ and $\mathrm{Pd} 4 p$ signals to be $2.7 \AA$.

Photoemission spectra were obtained using a hemispherical photoelectron energy analyzer, collecting electrons along the surface normal and at $40^{\circ}$ to the x-ray beam. Magnetic fields of up to $0.35 \mathrm{~T}$, either parallel or perpendicular to the sample surface, could be applied using a set of maneuverable in situ electromagnets and a pulsed power supply delivering up to 230 A. During the XPS measurements, the coils were earthed and removed from the vicinity of the sample. The experimental geometries of the MLDAD and MCDAD experiments are illustrated in Fig. 1. The combined energy resolution of the analyzer and monochromator was $0.4 \mathrm{eV}$.

\section{RESULTS AND DISCUSSION}

Fe $3 p$ XPS spectra for an Fe cluster-assembled film of thickness 2.8 ML (60 $⿱$ ) are shown in Fig. 2a. The lower two spectra were recorded under the MLDAD configuration, after applying a $\pm 0.35 \mathrm{~T}$ magnetic field pulse along the plane of the sample. The upper two spectra were recorded under the MCDAD geometry, after applying a $\pm 0.35 \mathrm{~T}$ magnetic field pulse in the out-of-plane direction. A clear change in the line shape of the $\mathrm{Fe} 3 p$ peak is observed in the former case upon reversal of the magnetic field. No such shift is apparent in the latter case.

The differences in intensity between spectra measured after reversing the applied field direction are shown in Fig. $2 \mathrm{~b}$. For the MLDAD measurements, two lobes of opposite sign are observed on either side of the peak, of the XPS

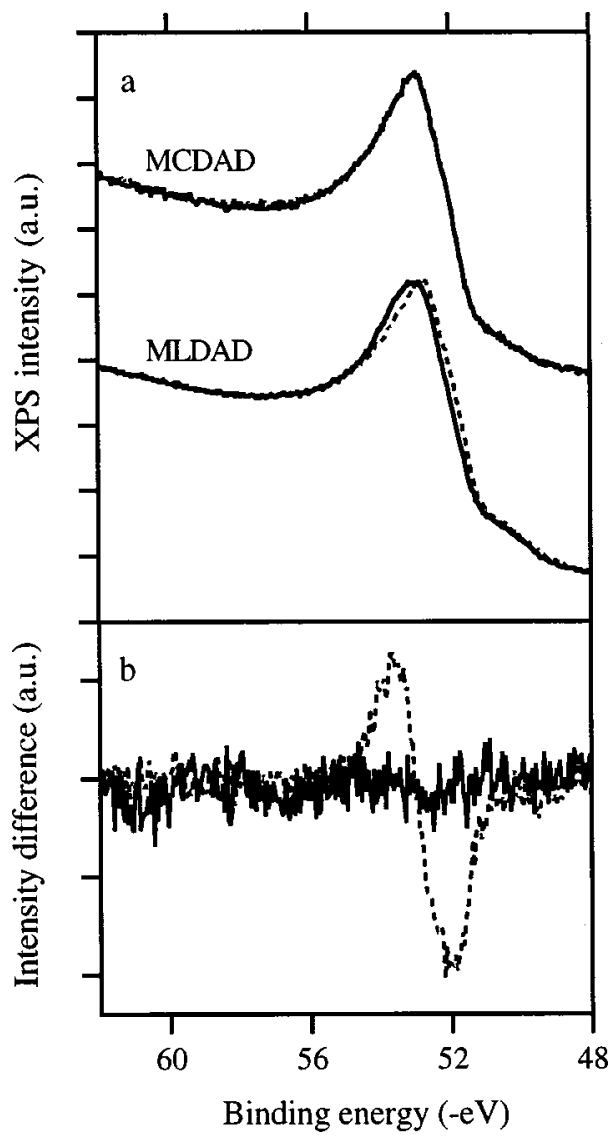

FIG. 2. (a) Fe $3 p$ XPS spectra for a 60 -Å-thick Fe cluster-assembled film, measured under the MCDAD geometry (top) and the MLDAD geometry (bottom); the solid and broken lines are measured after applying magnetic fields in opposite directions. (b) Difference spectra for MCDAD (solid line) and MLDAD (broken line) measurements.

spectrum, consistent with previous observations of magnetic dichroism in Fe $3 p$ photoemission. ${ }^{6-9}$ For the MCDAD measurements, no features are visible above the noise level in the difference spectrum.

A quantitative value for the magnetic dichroism signal can be obtained from the magnitude of the two features of the difference spectrum by normalizing the magnitude of their areas to the total area of the $\mathrm{Fe} 3 p$ photoemission peak, so that the signal is measured on a per atom basis. Then, multiplying the MLDAD signal by a factor of 0.3 accounts for the difference in the MLDAD and MCDAD intensities owing to the specific geometries in which the measurements are made, using the expressions for the geometrical dependence of the intensities presented in Ref. 12. The factor also corrects for the incomplete circular polarization of the x-ray beam.

The dependence of the Fe $3 p$ magnetic dichroism signal on the Fe cluster film thickness at a temperature of $40 \mathrm{~K}$ is shown in Fig. 3(a). For thicknesses below around half a monolayer, the MLDAD signal is zero within the experimental error. A small but finite MLDAD signal is observed between 0.5 and 1 ML thickness, followed by a steep rise between $1 \mathrm{ML}$ and 1.4 ML. Although the size of the MLDAD signal increases with coverage, no differences in the shape of either the $\mathrm{Fe} 3 p$ MLDAD difference spectrum or the 


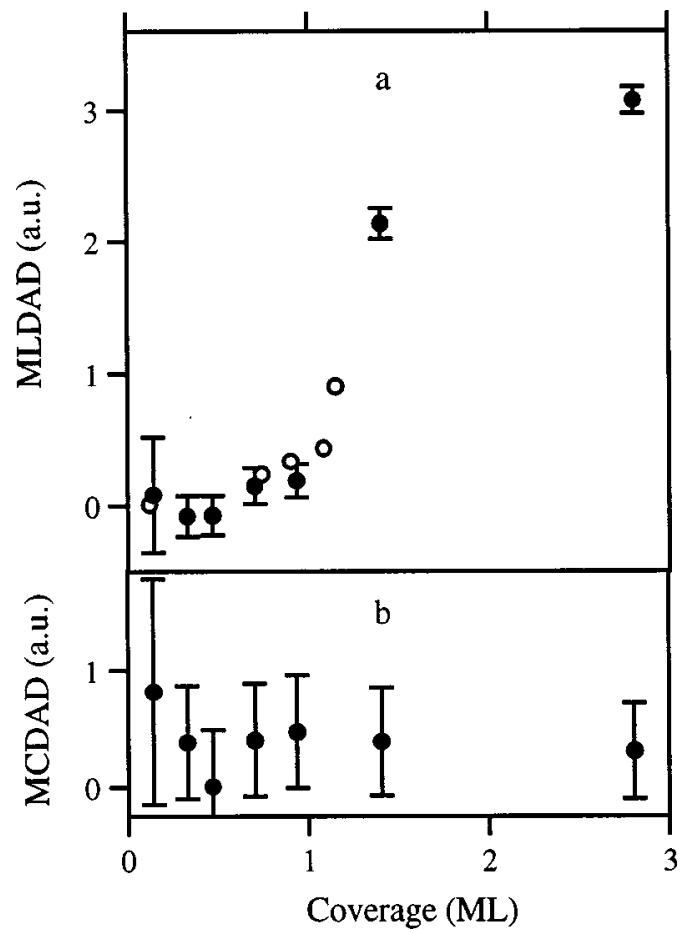

FIG. 3. (a) Fe $3 p$ MLDAD signal (for in-plane magnetization) vs coverage in monolayers of clusters, for $\mathrm{Fe}$ clusters deposited on $\mathrm{Cu}$ (filled circles), as well as for Fe clusters deposited on graphite (open circles, from Ref. 13). (b) $\mathrm{Fe} 3 p$ MCDAD signal (for out-of-plane magnetization), vs coverage of $\mathrm{Fe}$ clusters on $\mathrm{Cu}$.

magnetization-averaged $\mathrm{Fe} 3 p$ XPS spectrum are visible within the noise level for the different thicknesses. Meanwhile, no MCDAD signal is visible above the noise level for any of the films studied at this temperature.

The thickness dependence of the MLDAD response is reported elsewhere for nanoscale $\mathrm{Fe}$ clusters supported on highly oriented pyrolytic graphite, ${ }^{13}$ and these data are also presented in Fig. 3(a). In this reference, the cluster size distributions were similar to those considered here, although a surface oxide component was identified in the Fe $3 p$ spectrum, which is not seen for the present samples due to the reduced deposition time and the improved vacuum in the vicinity of the sample. In spite of the differences in the substrate and the purity of the clusters, similar behavior is observed: no measurable dichroism for isolated clusters, a small but finite MLDAD signal between 0.5 ML and $1 \mathrm{ML}$, and a rapid rise as the coverage is increased past $1 \mathrm{ML}$.

Previous scanning tunneling microscopy studies have indicated that the size distribution in the cluster beam is preserved upon deposition. ${ }^{11}$ Therefore, at low coverages, the deposited film is made up of isolated particles, average size $\approx 2.2 \mathrm{~nm}$, each consisting of a single magnetic domain. $\mathrm{X}$-ray magnetic circular dichroism studies of isolated $2.2 \mathrm{~nm}$ Fe particles have yielded a superparamagnetic blocking temperature, $T_{B}$, between $6 \mathrm{~K}$ and $9 \mathrm{~K}_{;}^{4}$ therefore, the Fe particles are superparamagnetic at a sample temperature of 40 $\mathrm{K}$. The thermal relaxation of the magnetization of the particles after removal of the applied field is fast compared to the measurement time $(\approx 10 \mathrm{~min}$.), and so no magnetic dichroism effects are observed, for either in-plane or out-of-

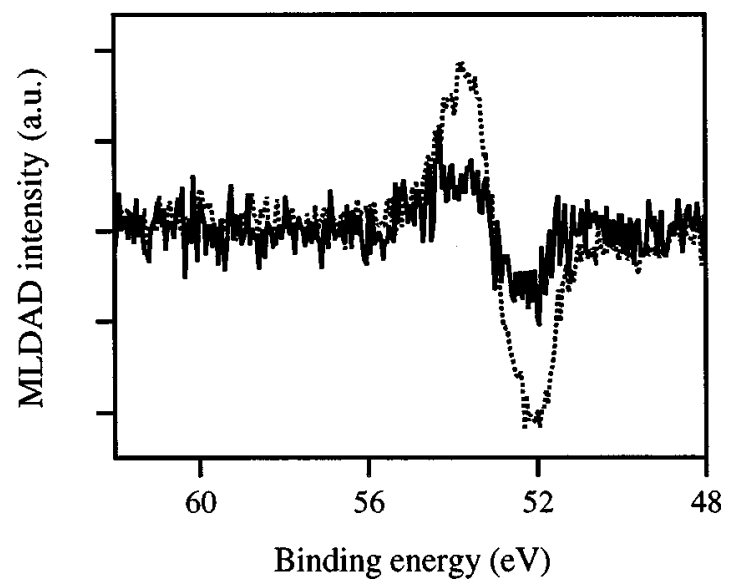

FIG. 4. Fe $3 p$ MLDAD difference spectra for exposed (broken line) and Pd capped (solid line) 60- $\AA$-thick Fe cluster-assembled films on $\mathrm{Cu}$.

plane applied fields. As the coverage increases, the interactions between the clusters lead to a stabilization of the magnetization at $40 \mathrm{~K}$; however, this produces strong out-ofplane demagnetizing fields, and so the remanent magnetization of the sample is in plane due to the shape anisotropy.

Magnetic circular dichroism measurements in x-ray absorption of Fe nanoclusters supported on graphite carried out at $10 \mathrm{~K}$ have revealed an increasing out-of-plane remanent magnetization with increasing film thickness, ${ }^{3}$ in contrast to the present result. In that study the sample temperature was close to the blocking temperature for isolated $2.2 \mathrm{~nm} F$ clusters and the increasing out-of-plane magnetization was ascribed to an increase in the effective particle size due to exchange coupling between the clusters, leading to an increase of $T_{B}$. In the present experiment, the sample temperature of $40 \mathrm{~K}$ is too high to observe a large stable magnetization within the measurement time for coverages below around $1 \mathrm{ML}$. Above this thickness, the out-of-plane direction is the hard magnetic axis due to shape anisotropy, and only an in-plane remanent magnetization can be detected.

We also observed the influence of a Pd capping layer on the magnetism of the Fe films. Figure 4 shows MLDAD difference spectra for the 2.8 ML Fe cluster-assembled film, before and after capping with a Pd film of thickness $2.7 \AA$. These spectra are normalized to the same intensity of the magnetization averaged $\mathrm{Fe} 3 p$ XPS. It can be seen that the Pd capped sample displays a much smaller MLDAD response (per atom) than the exposed film, yet, within experimental errors, the shape of the difference spectrum (the positions of the peaks and the ratio of height to width) is unaffected by the presence of the capping layer. The $\mathrm{Pd} 4 p$ core level, which partially overlaps with the Fe $3 p$ level, does not contribute to the difference spectrum.

The integrated MLDAD intensity is smaller by a factor of 3 in the capped sample. The MCDAD signal is zero within the experimental error for both the capped and exposed samples; therefore, the reduced MLDAD response is not due to a switching of the easy axis of magnetization from in plane into a perpendicular orientation. A similar reduction of the magnetic dichroism response has been observed elsewhere in x-ray photoemission of Fe films capped with a 1 
ML of $\mathrm{Pd},{ }^{14}$ and is ascribed to the influence of the $\mathrm{Fe} / \mathrm{Pd}$ interface upon the magnetism of the film.

The observed dichroism must come from Fe regions where there is no $3 d-4 d$ hybridization since this would have a marked effect on the shape of the difference spectrum, which is not observed. This indicates that the Fe regions in contact with the Pd are not magnetic and the only measurable dichroism originates from subsurface $\mathrm{Fe}$ atoms, or regions of the surface where the capping layer is not present due to uneven coverage. Alternatively, the Pd layer may modify the domain structure of the film, resulting in a decrease of the remanent magnetization.

${ }^{1}$ H. A. Dürr, G. van der Laan, J. Vogel, G. Panaccione, N. B. Brookes, E. Dudzik, and R. McGrath, Phys. Rev. B 58, 11853 (1998).

${ }^{2}$ H. A. Dürr, S. S. Dhesi, E. Dudzik, D. Knabben, G. van der Laan, J. B. Goedkoop, and F. U. Hillebrecht, Phys. Rev. B 59, 701 (1999).

${ }^{3}$ K. W. Edmonds, C. Binns, S. H. Baker, S. C. Thornton, C. Norris, J. B. Goedkoop, M. Finazzi, and N. B. Brookes, Phys. Rev. B 60, 472 (1999).
${ }^{4}$ K. W. Edmonds, C. Binns, S. H. Baker, M. J. Maher, S. C. Thornton, O. Tjernberg, and N. B. Brookes, Phys. Rev. B (submitted).

${ }^{5}$ P. Melinon, V. Paillard, V. Dupuis, A. Perez, P. Jensen, A. Hoareau, J. P. Perez, J. Tuaillon, M. Broyer, J. L. Vialle, M. Pellarin, B. Baguenard, and J. Lerme, Int. J. Mod. Phys. B 9, 339 (1995).

${ }^{6}$ F. Sirotti, G. Panaccione, and G. Rossi, Phys. Rev. B 52, 17063 (1995).

${ }^{7}$ S. H. Baker, K. W. Edmonds, A. M. Keen, S. C. Thornton, C. Norris, and C. Binns, Phys. Rev. B 61, 5026 (2000).

${ }^{8}$ Ch. Roth, F. U. Hillebrecht, H. B. Rose, and E. Kisker, Phys. Rev. Lett. 70, 3479 (1993)

${ }^{9}$ L. Baumgarten, C. M. Schneider, H. Petersen, F. Schäfers, and J. Kirschner, Phys. Rev. Lett. 65, 492 (1990).

${ }^{10}$ S. H. Baker, S. C. Thornton, K. W. Edmonds, M. J. Maher, C. Norris, and C. Binns, Rev. Sci. Instrum. (submitted).

${ }^{11}$ M. D. Upward, B. N. Cotier, P. Moriarty, P. H. Beton, S. H. Baker, C. Binns, and K. Edmonds, J. Vac. Sci. Technol. (accepted).

${ }^{12}$ G. van der Laan, Phys. Rev. B 51, 240 (1995).

${ }^{13}$ K. W. Edmonds, S. H. Baker, S. C. Thornton, M. J. Maher, A. M. Keen, and C. Binns, J. Appl. Phys. 86, 2651 (1999).

${ }^{14}$ X. Le Cann, C. Boeglin, B. Carrière, and K. Hricovini, Phys. Rev. B 54, 373 (1996). 